\title{
Intravenous Administration of Granulocyte-Colony Stimulating Factor for Stem Cells Mobilization and Third Degree Burn Wound Healing in Rats
}

\author{
Ali Samadikuchaksaraei ${ }^{1,2,3}$, Nahid Nasiri ${ }^{4,5}$, Mohammad Jafar Sharifi ${ }^{6^{*}}$, Narendra Pal Singh Chauhan ${ }^{7}$, Behrouz \\ Farhadihosseinabadi ${ }^{8,9}$, Sina Fatemi Sovini ${ }^{3}$, Mazaher Gholipourmalekabadi ${ }^{12^{*}}$ \\ ${ }^{1}$ Cellular and Molecular Research Center, Iran University of Medical Sciences, Tehran, Iran \\ ${ }^{2}$ Department of Tissue Engineering \& Regenerative Medicine, Faculty of Advanced Technologies in Medicine, Iran University of \\ Medical Sciences, Tehran, Iran \\ ${ }^{3}$ Department of Medical Biotechnology, Faculty of Allied Medicine, Iran University of Medical Sciences, Tehran, Iran \\ ${ }^{4}$ Department of Medical Laboratory Sciences, School of Paramedical Sciences, Shiraz University of Medical Sciences, Shiraz, Iran \\ ${ }^{5}$ Diagnostic Laboratory Sciences and Technology Research Center, School of Paramedical Sciences, Shiraz University of Medical \\ Sciences, Shiraz, Iran \\ ${ }^{6}$ Department of Immunology, Isfahan University of Medical Sciences, Isfahan, Iran \\ 'Department of Chemistry, Faculty of Science, Bhupal Nobles' University, Udaipur313002, Rajasthan, India \\ ${ }^{8}$ Biotechnology Department, School of Advanced Technologies in Medicine, Shahid Beheshti University of Medical Sciences, Tehran, \\ Iran \\ ${ }^{9}$ Student Research Committee, School of Medicine, Shahid Beheshti University of Medical Sciences, Tehran, Iran
}

Corresponding Authors: Mohammad Jafar Sharifi, Department of Immunology, Isfahan University of Medical Sciences, Isfahan, Iran. Email: mjsharifi63@yahoo.com

Mazaher Gholipourmalekabadi, Department of Tissue Engineering \& Regenerative Medicine, Iran University of Medical Sciences, Hemmat Highway, Tehran 144961-4535, Iran. Tel: +98-21-8862 4835, Email: mazaher.gholipour@iums.ac.ir;

mazaher.gholipour@gmail.com

Received January 4, 2019; Accepted May 21, 2019; Online Published September 11, 2019

\begin{abstract}
Introduction: Burns are one the most common skin damages which require medical intervention to be fully-recovered. In this light, tissue engineering field presents a vide verity of strategies including both scaffold-based and cell-based approaches to recover the damaged site.

Materials and Methods: In this study, the effects of granulocyte-colony stimulating factor (G-CSF) administration on mobilization of the bone marrow mesenchymal stem cells (MSCs) into defect area and treatment of the skin burn wound was examined in vivo. The G-CSF was injected intravenously into rats subjected to third degree burn wound. At days 3, 5, 7, 15 and 30 post-injections, the defect site was removed and investigated by H\&E and Malory's trichrome staining. The number of MSCs in blood samples was also determined by flow cytometry assay. Results: According to the results, intravenously administration of G-CSF significantly increased collagenesis and number of fibroblast cells infiltrated into the burned site, while decreased the severity of acute inflammatory response and amount of inflammatory cells comparing to control. The number of MSCs in bloodstream, representing the rate of MSCs migration, showed a 4-fold increase in the experimental group compared to control. Conclusions: The current study suggests the potential of intravenously administration of G-CSF as an effective strategy for treatment of severe burn injuries.

Keywords: Burn, Granulocyte-Colony Stimulating Factor (G-CSF), Mobilization, Wound Healing, Mesenchymal Stem Cell

Citation: Samadikuchaksaraei A, Nasiri N, Sharifi MJ, Chauhan NPS, Farhadihosseinabadi B, Fatemi Sovini S, Gholipourmalekabadi M. Intravenous administration of granulocyte-colony stimulating factor for stem cells mobilization and third degree burn wound healing in rats. J Appl Biotechnol Rep. 2019;6(3):83-87. doi:10.29252/JABR.06.03.01.
\end{abstract}

\section{Introduction}

Skin burn injury is considered as a critical clinical issue that profoundly affects the life quality of patients. ${ }^{1-3}$ It has been reported that about 2 million people are burned annually in the United States, among which around 80000 are hospitalized and 6500 of them die. ${ }^{4}$ The healing process of skin in burn injuries depending on the degree and severity of the damage, ranges from 3 (in superficial partial-thickness burns) to several weeks (in deep partial-thickness and full-thickness burns) (4-8). Burn wound healing is a complicated process that involves many cellular and molecular mechanisms and is orchestrated by cytokines and growth factors secreted from cells (such as macrophage and neutrophils) into the injured area. These factors affect the proliferation and migration of keratinocytes and fibroblasts. ${ }^{9-11}$

Epidermal growth factor (EGF), vascular endothelial 
growth factor (VEGF), transforming growth factor beta (TGF- $\beta$ ) and granulocyte-colony stimulating factor (G-CSF) are major cytokines and growth factors involved in this process. ${ }^{12}$ Among those, G-CSF is a cytokine which specifically stimulates differentiation of bone marrow progenitor cells to granulocytes and macrophages. ${ }^{13}$ The G-CSF also, stimulates proliferation of keratinocytes in an autocrine manner which results in regeneration of damaged skin. ${ }^{14}$ In vitro studies have shown that G-CSF enhances re-epithelialization through increasing the proliferation of keratinocytes. ${ }^{12}$ In addition, it has been demonstrated that G-CSF augments the migration and proliferation of endothelial and bone stromal cells from bone marrow into blood stream. ${ }^{15}$ Taken together, G-CSF plays a crucial role in burn wound healing as a cytokine. ${ }^{9,15}$ To fully treat third degree burn wounds, early dermabrasion and escharectomy followed by covering with skin grafts are recommended. ${ }^{816}$ Stem cell therapy has been established to overcome the obstacles associated with the application of skin substitutes such as difficulty and expensiveness. ${ }^{17}$

Stem cells, especially bone marrow-derived ones, have been found to be a promising cell source for treating burn injuries in recent decades. Easy accessibility and differentiation capabilities to a variety of cell types such as blood vessel cells, perifollicular cells, perisebaceous gland cells, keratinocytes and fibroblasts, which all play vital roles in the healing process, have made stem cells a potential source for cell therapy. ${ }^{18}$ It has been shown that efficiency of burn healing with mesenchymal stem cells (MSCs) transplantation is higher than other types like, fetal fibroblast cells. Nevertheless, lack of telomerase activity, low maintenance and proliferation rate of these cells in vitro have limited the applications of such cells. ${ }^{19}$ Regarding the fact that the G-CSF may affect the mobilization of MSCs from bone marrow, in the current study, this cytokine has been administrated intravenously in the third-degree burn wound model of rats and then its effects were evaluated on MSCs infiltration and wound healing in vivo.

\section{Materials and Methods}

In Vivo Study

The current study was performed on 40 adult male SpragueDawley rats (weighing 180-220 g) purchased from Pasteur Institute of Iran and acclimatized for one week under standard conditions prior to the experimental study. The rats were anesthetized by an IP injection ( $5 \mathrm{mg} / \mathrm{kg}$ body weight) of a 4:1 solution of ketamine hydrochloride and xylazine (Sigma, USA). After a general anesthesia induction, the back skins of animals were shaved by an electric clipper and then, exposed directly to boiling water $\left(98^{\circ} \mathrm{C}\right)$ for 30 seconds. The leather cover was used in order to avoid burning of uninvolved areas. ${ }^{20}$ The rats were randomly divided into 2 experimental groups: in the first group, the burned animals were treated with G-CSF and in the second one, normal saline solution was applied (each group containing 5 rats). After burning, the animals were intravenously administrated with G-CSF and normal saline for 9 consecutive days. ${ }^{21}$ The study was performed according to the guiding principles in the care and use of animals (Ethical code: IR.TUMS.REC 1390.926).22
Flow Cytometric Analysis

The flow cytometric analysis was performed to determine the mobilization rate of the MSCs from bone marrow into the blood stream. Half an hour after the last dose of G-CSF and normal saline injection, a 5-mL blood sample was taken from each animal. The mononuclear cells were isolated from the blood samples using ficoll (Sigma, USA). The isolated cells were then incubated with monoclonal anti-CD90 (FITC, Santa Cruz) and monoclonal anti-CD106 (PE, Santa Cruz) for 30 minutes. Anti IgG1-PE (Santa Cruz) and IgG1-FITC (Santa Cruz) served as negative controls. The population of CD90 and CD106 positive cells, representing the MSCs, were measured by flow cytometry (Facscaliber, Becton Dickinson). ${ }^{23}$

\section{Histological Analysis}

Histological observations were carried out 3, 5, 7 and 30 days after drug administration. To this end, five rats were randomly selected from each group. The histological specimens were collected from different areas of the burned site using a biopsy punch. The samples were fixed in $10 \%$ formalin, dehydrated through a series of ethanol concentrations $(70 \%, 80 \%, 90 \%$, $100 \%$ in distilled water), followed by embedding in xylene and paraffin. The samples were then sectioned serially $(8 \mu \mathrm{m}$ in thickness) by a microtome (Rotary microtome, MICRO DS 4055). The sections were stained with Harris Hematoxylin (EM Science) and eosin Y (Sigma, USA), and Malory's trichrome for histological analysis. ${ }^{24,25}$ The rate of collagen synthesis, the presence of inflammatory cells and number of fibroblast in the defected area were the major criteria evaluated in this study.

\section{Statistical Analysis}

The independent sample $t$ test was used for statistical data analysis. A $P$ value of $\leq 0.05$ was defined as the level of significance.

\section{Results}

Flow Cytometric Analysis

According to the obtained results from the flow cytometry assay, the number of $\mathrm{CD} 160^{+} / \mathrm{CD} 90^{+}$cells in peripheral blood significantly increased (approximately 4 -fold) in the G-CSF group compared to the control group (Figure 1). The average number of MSCs in the experimental and control groups was 624 cells $/ \mathrm{mL}$ and 155 cells $/ \mathrm{mL}$, respectively.

\section{Histological Analysis}

The histological observations revealed that the burn wound healing in the experimental group was remarkably faster than those in the control group (Figures 2 and 3).

The microscopic observations showed that the G-CSF administration significantly affected the number of fibroblast cells in the burned area of the experimental group in comparison to the control group. As a result, the inflammatory cells such as lymphocytes had a significant decrease in the G-CSF group compared to the control group by day 15 postadministration (Figure 2). The average number of fibroblast cells in the G-CSF group showed a 4.5 -fold increase in 
comparison to the control group after day 15 (28.8 and 6.4 cells in experimental and control, respectively) $(P \leq 0.01)$ (Figure 4).

Figure 3 shows the active fibroblast cells (black arrow) and collagen fibers in the control and collagen fibers and fibrocyte cells in the experimental samples, respectively. It is observable that, the G-CSF administration positively increased the (a)

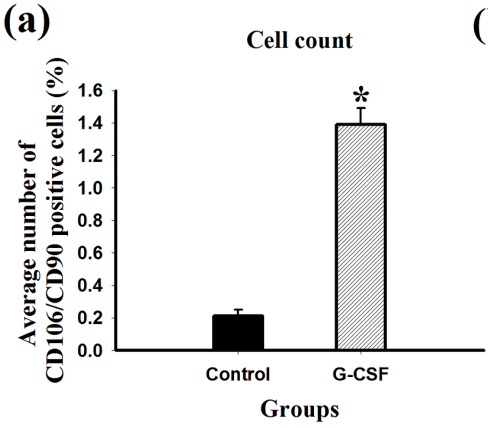

(b)

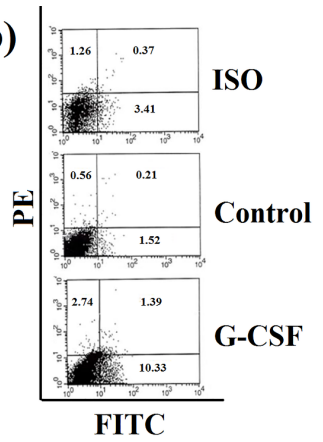

Figure 1. There was a 4 -fold increase in number of MSCs in experimental group (average $1.39 \%$ ) compared to control (average $0.21 \%$ ) (a). The flow cytometry results indicate the number of CD106 (PE) and CD90 (FITC) positive cells among the mononuclear cells isolated from the blood samples of rats (b). Asterisk indicates a significant difference with control $(P$ value $\leq 0.001)$
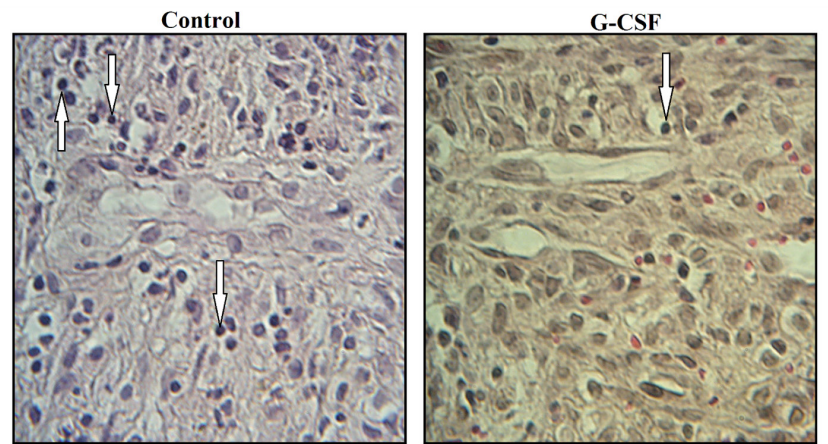

Day 15

Figure 2. The number of inflammatory cells (lymphocytes) was compared between H\&E-stained specimens of the control and experimental group on day 15 . Number of these cells decreased remarkably in the G-CSF group in comparison with control. Arrows show inflammatory cells. $\times 10$ magnification.

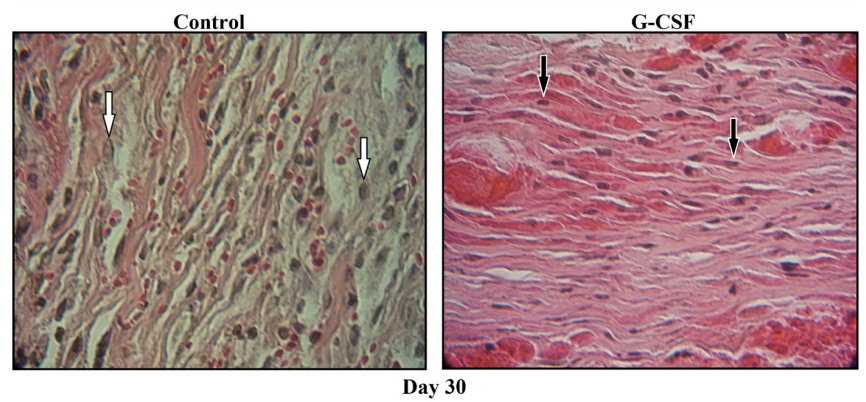

Figure 3. H\&E Stained Samples of the G-CSF and Control Groups on Day 30. A large numbers of active fibroblast cells (white arrow) and a few numbers of collagen fibers (red fibers) were observed in the control samples. In the G-CSF sample, the fibrocyte cells (black arrow) and collagen fibers (red fibers) were observed numerously compared to control. $\times 10$ magnification. fibroblast infiltration and collagenesis in the defect area. The Malory's Trichrome stained samples showed a remarkable collagenesis increase in the experimental group compared to the control group after days 15 and 30 (Figure 5). As it is shown in Figure 5, the collagenesis in G-CSF administrated group was clearly observed by day 15 .

\section{Discussion}

The efficiency and therapeutic applications of bone marrowderived stromal cells in soft tissue engineering, especially skin, have been reported by several studies. ${ }^{26-28}$ In this line of research, Mansilla et al have found that the number of these cells will increase in peripheral blood after burn injuries. ${ }^{27}$ Additionally, several studies have demonstrated that both migrations of stem cells and regeneration of damaged tissues are mediated by some growth factors such as G-MCSF. In

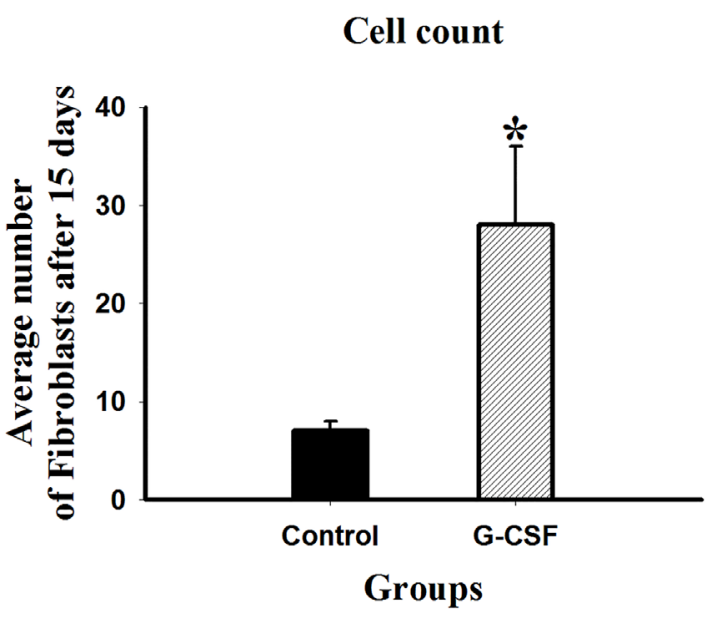

Figure 4. The Number of Fibroblast Cells in Defect Area on Day 15. The fibroblasts showed a 4.5 -fold increase in the G-CSF compared to control group. Asterisk indicates a significant difference with control $(P$ value $\leq 0.001)$.
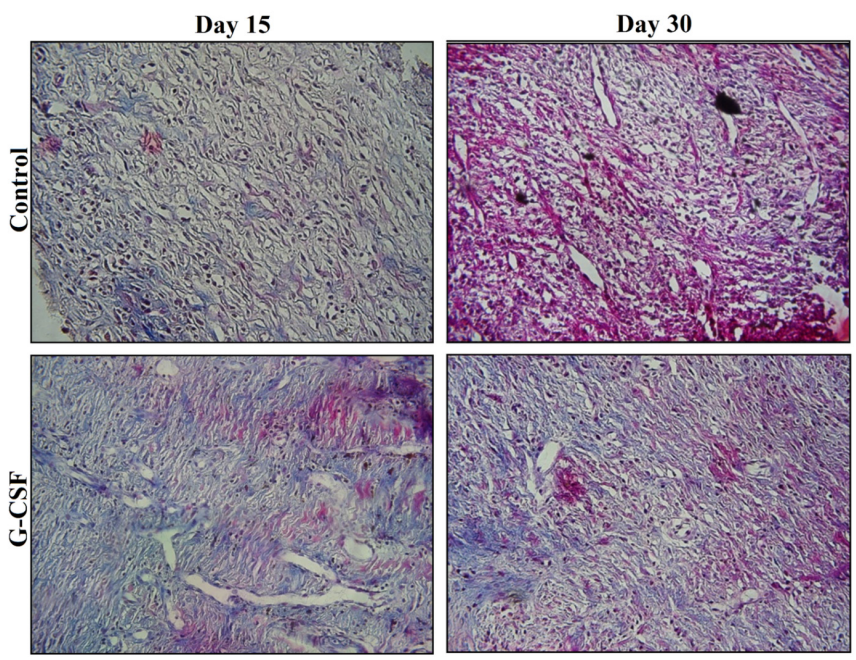

Figure 5. The Comparison of the Collagenesis Between the Malory's Trichrome Stained Samples of the G-CSF and Control Groups After Days 15 and 30. As it is clearly observable, the collagenesis remarkably increased in the G-CSF group compared to control in both days 15 and 30. $\times 40$ magnification. 
the current study, the role of G-CSF administration has been represented on the mobilization of bone marrow-derived stromal stem cells into the peripheral blood and its healing potential of third-degree burn injury created in rats.

The results obtained from this study revealed that the administration of the G-CSF significantly increases the number of MSCs in peripheral blood 4 -fold $(P \leq 0.05)$. These results were consistent with other studies conducted in this area. ${ }^{19,21,29,30}$ The mechanisms by which the G-CSF affects the migration of MSCs are not fully understood, but seems to be associated with SDF-1/CXCR4 ligand/receptor pair. The SDF-1, as a chemokine, and its receptor CXCR4 are found to have an essential role in stem cells homing. ${ }^{31}$ It has been reported that G-CSF reduces the concentration of SDF-1 in bone marrow and leads to release the progenitor cells into the peripheral blood. ${ }^{32}$ The histological observations showed a decrease in the number of inflammatory cells in the experimental samples compared to the control samples 15 days post-injection. According to other studies, these findings could be due to the anti-inflammatory role of mesenchymal stem cells. These results were in line with those obtained from Shen et al's ${ }^{33}$ study in which locally administration of G-CSF in the rat full-thickness wounds significantly decreased the inflammatory cells within the wound bed.

The mesenchymal cells can eventually differentiate to various cell types such as collagen producing fibroblasts. ${ }^{34,25}$ The present study showed a significant difference in the number of fibroblasts among experimental and control samples $(P \leq 0.01)$ in day 15 . The MSCs are involved in the formation of extracellular matrix (collagen type I, IV and fibronectin) through secretion of numerous cytokines and growth factors and thereby provide a microenvironment to support cell growth and maturation, especially in damaged tissues. Chen et al showed the potential of MSCs in secretion of some paracrine factors that are associated with the repair of damaged tissues. ${ }^{36}$ In the present study, the collagen fibers were clearly observed in biopsy specimens of the test group by day 15 post injection. The higher collagenesis rate in the experimental group was quite evident in comparison to the control. Additionally, the G-SCF group showed a decreased cellular infiltration and increased number of fibroblast cells when compared with control samples. The therapeutic role of the G-CSF in this study may not only be due to the increase of bone marrow-derived cells infiltration, but also results from its effects on the migration of endothelial precursors and hematopoietic stem cells into defect area. All of these cells are responsible for angiogenesis and wound healing through secretion of angiogenic factors such as VEGF, bFGF and Ang$1 .{ }^{37}$ On the other hand, the role of G-CSF in induction of endothelial cell proliferation has been previously reported. ${ }^{38}$ Furthermore, the G-CSF administration on cardiac defects found to increase the expression of the adherent molecules such as ICAM-1 at the site of damaged blood vessels as well as the infiltration of MSCs. ${ }^{39}$ Taken together, the data of this study strongly demonstrates the positive effects of G-CSF on migration of bone marrow-derived cells and eventually, healing the skin lesions, that is concordant with other studies that showed the role of MSCs in skin injuries healing without using the G-CSF. ${ }^{18,27,28,36}$

\section{Conclusions}

The increasing importance of cell therapy in burn wound healing and the necessity of developing a safe and effective therapeutic method, has attracted the attention of researchers for designing an alternative strategy to minimize the challenges associated with stem cell therapy such as rejection and low proliferation rate. Recently, researchers have focused on the application of growth factors involved in tissue regeneration rather than stem cells as an alternative strategy in cell therapy, in damaged tissue repair. In this line of research, the efficacy of G-CSF administration in MSCs migration and burn injury healing in rats was examined in this study. All the collected data clearly indicated the potential role of the G-CSF in MSCs infiltration to the defect site and third-degree burn wound healing. Therefore, this study can serve as a pilot to further investigate the therapeutic abilities of the G-CSF in soft tissue engineering.

\section{Authors' Contributions}

MG, AS, NN and MJS designed the concept of the study. MG wrote the manuscript. NP, AS and NN equally performed the experiments. BF revised the manuscript. AS and NN contributed equally to this research.

\section{Conflict of Interest Disclosures}

The authors declare no conflict of interest neither in terms of financial issues nor in regards to the publication of this study.

\section{Acknowledgments}

We would like to show our deepest gratitude to the staff of tissue engineering department of Iran University of Medical Sciences.

\section{References}

1. Mohammadi AA, Sabet B, Riazi H, Tavakkolian AR, Mohammadi MK, Iranpak S. Human amniotic membrane dressing: an excellent method for outpatient management of burn wounds. Iran J Med Sci. 2009;34(1):61-64.

2. Ravishanker R, Bath AS, Roy R. "Amnion Bank"--the use of long term glycerol preserved amniotic membranes in the management of superficial and superficial partial thickness burns. Burns. 2003;29(4):369-374. doi:10.1016/S0305-4179(02)00304-2.

3. Gholipourmalekabadi M, Mozafari M, Salehi M, et al. Development of a cost-effective and simple protocol for decellularization and preservation of human amniotic membrane as a soft tissue replacement and delivery system for bone marrow stromal cells. Adv Healthc Mater. 2015;4(6):918-926. doi:10.1002/ adhm.201400704.

4. Singh V, Devgan L, Bhat S, Milner SM. The pathogenesis of burn wound conversion. Ann Plast Surg. 2007;59(1):109-115. doi:10.1097/01.sap.0000252065.90759.e6.

5. Atiyeh BS, Gunn SW, Hayek SN. State of the art in burn treatment. World J Surg. 2005;29(2):131-148. doi:10.1007/s00268-0041082-2.

6. Barret JP, Dziewulski P, Ramzy PI, Wolf SE, Desai MH, Herndon DN. Biobrane versus $1 \%$ silver sulfadiazine in second-degree pediatric burns. Plast Reconstr Surg. 2000;105(1):62-65. doi:10.1097/00006534-200001000-00010.

7. Gholipourmalekabadi M, Bandehpour M, Mozafari M, et al. Decellularized human amniotic membrane: more is needed for an efficient dressing for protection of burns against antibiotic-resistant bacteria isolated from burn patients. Burns. 2015;41(7):14881497. doi:10.1016/j.burns.2015.04.015. 
8. Hosseini SN, Karimian A, Mousavinasab SN, Rahmanpour H, Yamini M, Zahmatkesh SH. Xenoderm versus $1 \%$ silver sulfadiazine in partial-thickness burns. Asian J Surg. 2009;32(4):234-239. doi:10.1016/s1015-9584(09)60400-0.

9. Hu X, Sun H, Han C, Wang X, Yu W. Topically applied rhGM-CSF for the wound healing: a systematic review. Burns. 2011;37(5):729741. doi:10.1016/j.burns.2010.08.016.

10. Fang $Y$, Shen J, Yao M, Beagley KW, Hambly BD, Bao S. Granulocyte-macrophage colony-stimulating factor enhances wound healing in diabetes via upregulation of proinflammatory cytokines. Br J Dermatol. 2010;162(3):478-486. doi:10.1111/ j.1365-2133.2009.09528.x.

11. Baker EA, Leaper DJ. Proteinases, their inhibitors, and cytokine profiles in acute wound fluid. Wound Repair Regen. 2000;8(5):392398. doi:10.1046/j.1524-475x.2000.00392.x.

12. Barrientos S, Stojadinovic O, Golinko MS, Brem H, TomicCanic M. Growth factors and cytokines in wound healing. Wound Repair Regen. 2008;16(5):585-601. doi:10.1111/j.1524475X.2008.00410.x.

13. Peters WP, Rosner G, Ross $M$, et al. Comparative effects of granulocyte-macrophage colony-stimulating factor (GM-CSF) and granulocyte colony-stimulating factor (G-CSF) on priming peripheral blood progenitor cells for use with autologous bone marrow after high-dose chemotherapy. Blood. 1993;81(7):17091719.

14. Imokawa G, Yada Y, Kimura M, Morisaki N. Granulocyte/ macrophage colony-stimulating factor is an intrinsic keratinocytederived growth factor for human melanocytes in UVA-induced melanosis. Biochem J. 1996;313 ( Pt 2):625-631. doi:10.1042/ bj3130625.

15. Mann A, Breuhahn K, Schirmacher P, Blessing M. Keratinocytederived granulocyte-macrophage colony stimulating factor accelerates wound healing: Stimulation of keratinocyte proliferation, granulation tissue formation, and vascularization. J Invest Dermatol. 2001;117(6):1382-1390. doi:10.1046/j.0022202x.2001.01600.x.

16. Gholipourmalekabadi M, Sameni M, Radenkovic D, Mozafari M, Mossahebi-Mohammadi M, Seifalian A. Decellularized human amniotic membrane: how viable is it as a delivery system for human adipose tissue-derived stromal cells? Cell Prolif. 2016;49(1):115121. doi:10.1111/cpr.12240.

17. Eroglu E, Agalar F, Altuntas I, Eroglu F. Effects of granulocytecolony stimulating factor on wound healing in a mouse model of burn trauma. Tohoku J Exp Med. 2004;204(1):11-16. doi:10.1620/ tjem.204.11.

18. Badiavas EV, Falanga V. Treatment of chronic wounds with bone marrow-derived cells. Arch Dermatol. 2003;139(4):510-516. doi:10.1001/archderm.139.4.510.

19. Lund TC, Tolar J, Orchard PJ. Granulocyte colony-stimulating factor mobilized CFU-F can be found in the peripheral blood but have limited expansion potential. Haematologica. 2008;93(6):908-912. doi:10.3324/haematol.12384.

20. Shumakov VI, Onishchenko NA, Rasulov MF, Krasheninnikov ME, Zaidenov VA. Mesenchymal bone marrow stem cells more effectively stimulate regeneration of deep burn wounds than embryonic fibroblasts. Bull Exp Biol Med. 2003;136(2):192-195. doi:10.1023/a:1026387411627.

21. Tatsumi K, Otani H, Sato D, et al. Granulocyte-colony stimulating factor increases donor mesenchymal stem cells in bone marrow and their mobilization into peripheral circulation but does not repair dystrophic heart after bone marrow transplantation. Circ J. 2008;72(8):1351-1358. doi:10.1253/circj.72.1351.

22. Tan B. Guidelines on the Care and Use of Animals for Scientific Purposes. National Advisory Committee for Laboratory Animal Research; 2004.

23. Samadikuchaksaraei A, Gholipourmalekabadi M, Erfani Ezadyar $\mathrm{E}$, et al. Fabrication and in vivo evaluation of an osteoblastconditioned nano-hydroxyapatite/gelatin composite scaffold for bone tissue regeneration. J Biomed Mater Res A. 2016;104(8):2001-
2010. doi:10.1002/jbm.a.35731.

24. Gholipourmalekabadi M, Mozafari M, Bandehpour $M$, et al. Optimization of nanofibrous silk fibroin scaffold as a delivery system for bone marrow adherent cells: in vitro and in vivo studies. Biotechnol Appl Biochem. 2015;62(6):785-794. doi:10.1002/ bab.1324.

25. Gholipourmalekabadi M, Mozafari M, Gholipourmalekabadi $M$, et al. In vitro and in vivo evaluations of three-dimensional hydroxyapatite/silk fibroin nanocomposite scaffolds. Biotechnol Appl Biochem. 2015;62(4):441-450. doi:10.1002/bab.1285.

26. François S, Mouiseddine M, Mathieu N, et al. Human mesenchymal stem cells favour healing of the cutaneous radiation syndrome in a xenogenic transplant model. Ann Hematol. 2007;86(1):1-8. doi:10.1007/s00277-006-0166-5.

27. Mansilla E, Marin GH, Sturla F, et al. Human mesenchymal stem cells are tolerized by mice and improve skin and spinal cord injuries. Transplant Proc. 2005;37(1):292-294. doi:10.1016/j. transproceed.2005.01.070.

28. Rasulov MF, Vasilchenkov AV, Onishchenko NA, et al. First experience of the use bone marrow mesenchymal stem cells for the treatment of a patient with deep skin burns. Bull Exp Biol Med. 2005;139(1):141-144. doi:10.1007/s10517-005-0232-3.

29. Goldberg ED, Dygai AM, Zyuz'kov GN, Zhdanov VV. Mechanisms of mobilization of mesenchymal precursor cell under the effect of granulocytic colony-stimulating factor and hyaluronidase. Bull Exp Biol Med. 2007;144(6):802-805. doi:10.1007/s10517-0070435-x.

30. Ripa RS, Haack-Sørensen M, Wang Y, et al. Bone marrow derived mesenchymal cell mobilization by granulocyte-colony stimulating factor after acute myocardial infarction: results from the Stem Cells in Myocardial Infarction (STEMMI) trial. Circulation. 2007;116(11 Suppl):I24-30. doi:10.1161/circulationaha.106.678649.

31. Lapidot T, Kollet O. The essential roles of the chemokine SDF-1 and its receptor CXCR4 in human stem cell homing and repopulation of transplanted immune-deficient NOD/SCID and NOD/SCID/ B2m(null) mice. Leukemia. 2002;16(10):1992-2003. doi:10.1038/ sj.leu.2402684.

32. Son BR, Zhao D, Marquez-Curtis LA, Shirvaikar N, Ratajczak MZ, Janowska-Wieczorek A. SDF-1-CXCR4 and HGF-c-met Axes Regulate Mobilization/Recruitment to Injured Tissue of Human Mesenchymal Stem Cells. Blood. 2004;104(11):2331.

33. Shen GY, Park IH, Song YS, et al. Local injection of granulocytecolony stimulating factor accelerates wound healing in a rat excisional wound model. Tissue Eng Regen Med. 2016;13(3):297303. doi:10.1007/s13770-016-9054-9.

34. Pittenger MF, Mackay AM, Beck SC, et al. Multilineage potential of adult human mesenchymal stem cells. Science. 1999;284(5411):143-147. doi:10.1126/science.284.5411.143.

35. Oswald J, Boxberger S, Jørgensen B, et al. Mesenchymal stem cells can be differentiated into endothelial cells in vitro. Stem Cells. 2004;22(3):377-384. doi:10.1634/stemcells.22-3-377.

36. Chen L, Tredget EE, Wu PY, Wu Y. Paracrine factors of mesenchymal stem cells recruit macrophages and endothelial lineage cells and enhance wound healing. PLoS One. 2008;3(4):e1886. doi:10.1371/journal.pone.0001886.

37. Kinnaird T, Stabile E, Burnett MS, et al. Marrow-derived stromal cells express genes encoding a broad spectrum of arteriogenic cytokines and promote in vitro and in vivo arteriogenesis through paracrine mechanisms. Circ Res. 2004;94(5):678-685. doi:10.1161/01.res.0000118601.37875.ac.

38. Bussolino F, Wang JM, Defilippi P, et al. Granulocyte- and granulocyte-macrophage-colony stimulating factors induce human endothelial cells to migrate and proliferate. Nature. 1989;337(6206):471-473. doi:10.1038/337471a0.

39. Deindl E, Zaruba MM, Brunner S, et al. G-CSF administration after myocardial infarction in mice attenuates late ischemic cardiomyopathy by enhanced arteriogenesis. FASEB J. 2006;20(7):956-958. doi:10.1096/fj.05-4763fje. 